\title{
The effect of industrial processing of salmon oil on its ability to reduce serum concentrations of oxidized low-density lipoprotein- $\beta 2$-glycoprotein-I complex in a mouse model
}

\author{
Bomi Framroze $^{1}$ and Sagar Sawant ${ }^{2}$ \\ ${ }^{1}$ Hofseth Biocare ASA, Molvegen 6, Aalesund 6000, Norway; ${ }^{2}$ SA-FORD, MIDC Taloja, Navi \\ Mumbai, 410028, India
}

*Corresponding author: Bomi Framroze,PhD, CSO, Hofseth Biocare AS, Molovegen 6, 6000 Ålesund, Norway

Submission date: August 17, 2014; Acceptance date: October 29, 2014; Publication date: October 31, 2014

\begin{abstract}
:
Background: Circulating serum levels of oxidized low-density lipoprotein, $\beta 2$-glycoprotein I complex (oxLDL-GP), have been previously correlated with adverse cardiovascular events and have been shown to be reduced by consumption of enzymatically liberated extra virgin salmon oil (EVSO). This mouse study measured the changes in the oxLDL-GP lowering effect when consuming EVSO with varying levels of EPA+DHA (eicosapentenoic acid and docosahexenoic acid) as well as when consuming EVSO that was subjected to various processing treatments commonly carried out during fish oil production.
\end{abstract}

Methods: Sprague Dawley mice were fed a diet containing eight different EVSO's incorporated into a normal diet at the Human Equivalent Dose (HED) of $1000 \mathrm{mg}$ for 8 weeks. Serum was collected at the start and at the end of the trial and the oxLDL-GP concentrations were measured using an ELISA assay. Statistical analysis of the results was carried out using a 1-tail, paired Student t-Test.

Results: In order to lower circulatory oxLDL-GP levels, the mice had to consume a minimum of $80 \mathrm{mg}$ per day HED of EPA+DHA. Heat treatment of the EVSO did not affect this bioactivity but hydrolysis with acid or base and re-esterification to the triglyceride form or significant oxidation (rancidity) rendered the oil inactive on this important cardio-vascular disease (CVD) biomarker.

Conclusions: This result shows that harsh processing conditions on fish oils can lead to the destruction of biological efficacy in spite of increasing the concentration of typical fish oil bioactive constituents such as EPA+DHA. It also lends support to the developing nutrition theory that eating highly-refined, processed or concentrated-ingredient supplements derived from functional foods may not be able to reproduce their full nutritive and health-benefiting effects. 
Keywords: Oxidized-LDL; processing; Salmon; Oil; omega-3; rancidity

\section{BACKGROUND:}

Atherosclerosis is a multi-factor chronic pathologic process associated with significant cardiovascular morbidity and mortality. Over the past few years many trials have reported on the beneficial effects of eating fish in patients with preexisting CVD as well as in healthy individuals. [1] Zhang et al, [2] showed that fish consumption was associated with a reduced risk from all-cause, ischemic heart disease and stroke mortality across 36 countries. In the Chicago Western Electric Study, [3] men who consumed 35g of fish daily had a 50\% lower risk of death from coronary heart disease (CHD), as compared to the control group. Other epidemiological studies have also reported that men who ate at least some fish weekly had a lower (CHD) mortality rate compared to men who ate no fish in their diet. [4,5] The recent Nurses' Health Study [6] has shown an inverse association between fish intake and CHD death on a dosedependent curve. In another population-based, case-controlled study, a 50\% reduced risk of primary cardiac arrest was reported between fish intake and risk for sudden death with consuming only two oily fish meals per week. [7]

Some studies, such as the Health Professionals' Follow-up Study, [8] and the US Physicians' Health Study [9] have not shown a beneficial effect from fish intake (and omega-3 fatty acids) and risk of any CHD nor a reduced risk of total myocardial infarction (MI) or cardiovascular mortality, although, as appropriate to the conclusions of this paper, fish consumption alone was related with a reduced risk of total mortality. More significantly, this seeming lack of an association between fish intake (and omega-3 fatty acids) and CHD incidence and mortality was also seen in the EURAMIC (European Multicenter Case-Control Study on Antioxidants, Myocardial Infarction and Breast Cancer) Study [10] where no evidence of reduced risk of MI was seen in spite of increased levels of adipose tissue DHA. In fact, in extension to these studies that do not show a beneficial effect, the Alpha-Tocopherol, BetaCarotene Cancer Prevention Study found that estimated omega-3 fatty acid intake from fish was actually associated with a trend towards increased risk of coronary death when adjusted for fatty acid types.[11]

It is possible to arrive at some explanations for the conflicting data from the epidemiological studies due to the differences in definitions of sudden death, variable healthy lifestyle definitions, experimental design and test substances, different study populations and variability in the end points studied. [12] One explanation, based on an analysis of 11 studies, is that the protective effect of fish consumption relates to the CHD risk status of the population studied [13] and that fish consumption reduces CHD mortality in high-risk but not low-risk populations. The type of fish and associated lipid biofactors, besides omega-3's, may also play a role in observing changes is CVD. Oomen et al [14] reported a lower CHD mortality in populations that consumed fatty fish but not lean fish while other epidemiological studies have hypothesized on the adverse effects of methylmercury, an environmental contaminant found in certain fish that may diminish the health benefits of omega-3 fatty acids. [15] Another important fatty acid, $\alpha-$ linolenic acid, has recently shown significant effect on lowering the risk of MI and fatal ischemic heart disease. [16] 
Several epidemiological studies have also examined the relationship between fish intake and stroke incidence. Zutphen showed a lowering of the unadjusted hazard risk ratio for men who consumed an average of 20g/day of fish. [17] Yet again, in other studies like the Lyon Diet Heart Study [18] (testing a Mediterranean-style diet enriched with $\alpha$-linolenic acid) and the GISSIPrevention Study [19] (testing the effects of $850 \mathrm{mg}$ of supplemental omega-3 fatty acids), there was no significant effect on the incidence of stroke.

To assist in narrowing down the relationship between CVD and fish/fish oil intake many researchers have begun to study the dietary effect of fish/fish oil intake on specific serologic markers of inflammation that have been associated with cardiovascular disease (CVD) progression [20]. Friedman et al have shown that oral fish oil supplementation lowers C-reactive protein in serum as a potential indication of reduced inflammatory risk. [21] Recent clinical evaluations have shown that oxidized low-density lipoprotein is highly inflammatory with proatherogenic properties that promote endothelial dysfunction.[22] Damsgaard et al have shown that fish oil supplementation lowers plasma triglyceride levels but does not impact other serological CVD biomarkers like oxLDL. [23] Unlike native LDL, oxLDL binds in vitro to 32 glycoprotein I, a phospholipid-binding plasma protein, in a time- and temperature- dependent manner to form covalently bound stable oxidized low-density lipoprotein- $\beta 2$-2-glycoprotein-1 (oxLDL-GP) complexes. [24] The association of oxLDL-GP complexes with CVD severity and adverse outcomes in patients with associated inflammatory diseases has also been reported. [25]

We have previously shown that a daily dietary supplement of $1000 \mathrm{mg} /$ day of EVSO reduces circulatory levels of oxLDL-GP in healthy human subjects. This oxLDL-GP concentration lowering effect was not observed with standard 18/12 fish oil or the control vegetable oil and minimally observed with other highly processed oils such as krill and algae. [26]

The aim of this study is to examine the effect of varying the daily intake levels of the polyunsaturated fatty acids, eicosapentenoic acid (EPA), docosahexenoic acid (DHA), 1 and various manufacturing unit-process steps such as treatment with acid, base, heat, and oxidation on natural EVSO using an ELISA assay to measure circulating serum concentrations of the oxLDL-GP CVD biomarker in Sprague-Dawley mice. Our results may lend some clarity to the highly varied epidemiological results of dietary fish and fish oil intake on CVD, by studying the impact of specific fish oil manufacturing processing steps on the CVD marker, oxLDL-GP.

\section{METHODS \& MATERIALS:}

This study was conducted according to GCP guidelines issued by the ICH and CDSCO ethical guidelines in accordance with the laws and regulations of India where the trial was performed. The final approved protocol, informed consent documents, and all the study related documents were reviewed and approved by an Institutional Animal Ethics Committee before the start of the study.

Study Design: 64 healthy Swiss Albino mice (Mus Musculus) of equal gender (females were nulliparous and non-pregnant) and up to 10 weeks in age completed this 8 week randomized open label study. Animals were acclimatized to the test conditions for a minimum period of 5 days prior to the test. Prior to initiation of test item/placebo administration, the animals were randomly allocated to the eight different test groups (G1-G8) using the 'Group Allocation function in the MS Excel Add-in "Daniel's XL Toolbar," (http://xltoolbox.sourceforge.net/) in 
such a way that weight variation of animals was within $20 \%$ of group mean. The animals were marked individually by temporary tail marking with indelible ink and cage cards during acclimatization and by micro tattooing of toe pads, after randomization.

Following allocation to the study, each animal was assigned an individual cage card, labeled with species, strain, sex, project number, animal number, group number, dose level, and experiment start and end dates.

Corn cob from M/s. Sparconn Lifescience, was used as bedding material. The average room temperature and relative humidity of experimental room were maintained between 19 to $23^{\circ} \mathrm{C}$ and 43 to $55 \%$, respectively, during the entire study period. Artificial light was set to give a cycle of 12 hours light and 12 hours dark. A minimum of 12 air changes per hour were provided to the animal room. The animals were offered a conventional laboratory rodent diet ad libitum supplied by M/s. Nutrivet Life Sciences and water was provided ad libitum.

Test Formulations: Seven samples of EVSO were prepared with varying levels of EPA+DHA and different production treatments, as shown in Table 1 below.

Table 1. EPA/DHA composition and process treatment of test oils per group

\begin{tabular}{|c|c|}
\hline Group/Dose: & \\
\hline G1 & Sunflower oil / 206mg/kg/day (negative control) \\
\hline G2 & EVSO $80 \mathrm{mg} / \mathrm{g}$ EPA+DHA / 206mg/kg/day \\
\hline G3 & $\begin{array}{l}\text { EVSO } 40 \mathrm{mg} / \mathrm{g} \mathrm{EPA}+\mathrm{DHA} / 103 \mathrm{mg} / \mathrm{kg} / \mathrm{day}+103 \mathrm{mg} / \mathrm{kg} / \text { day of } \\
\text { sunflower oil }\end{array}$ \\
\hline G4 & EVSO $120 \mathrm{mg} / \mathrm{g}$ EPA+DHA / 206mg/kg/day \\
\hline G5 & EVSO 180mg/g EPA+DHA / 206mg/kg/day \\
\hline G6 & $\begin{array}{l}\text { EVSO } 120 \mathrm{mg} / \mathrm{g} \text { EPA+DHA after heat treatment / } \\
206 \mathrm{mg} / \mathrm{kg} / \text { day }\end{array}$ \\
\hline G7 & $\begin{array}{l}\text { EVSO } 120 \mathrm{mg} / \mathrm{g} \text { EPA+DHA after hydrolysis \& re-TG/ } \\
206 \mathrm{mg} / \mathrm{kg} / \text { day }\end{array}$ \\
\hline G8 & EVSO $120 \mathrm{mg} / \mathrm{g}$ EPA+DHA after air oxidation $/ 206 \mathrm{mg} / \mathrm{kg} /$ day \\
\hline
\end{tabular}

The mouse dose was selected based on the proposed human (60 kg weight) dose of the EVSO at $1000 \mathrm{mg} /$ day. Based on body surface area calculations, the mouse equivalent dose was determined to be $206 \mathrm{mg} / \mathrm{kg} /$ day and hence this was applied across all groups.

Mice in the Group G1 were fed a diet containing sunflower oil as the negative control. Mice in Group G2 were fed the natural EVSO containing $80 \mathrm{mg} / \mathrm{g}$ of EPA+DHA as the positive 
control group. In Group G3, the amount of EVSO was reduced by half to $103 \mathrm{mg} / \mathrm{kg} / \mathrm{day}$ and the remainder of the fatty acid quantity was made up with $103 \mathrm{mg} / \mathrm{kg} /$ day of sunflower oil so that the mice only received $40 \mathrm{mg} / \mathrm{kg} /$ day of EPA+DHA. To increase the daily dose of EPA+DHA to $120 \mathrm{mg} / \mathrm{kg} /$ day in Group G4 and to $180 \mathrm{mg} / \mathrm{kg} /$ day in Group G5, we used a selective lipase treatment $\left[{ }^{27}\right]$ to increase the relative EPA+DHA in the EVSO to $120 \mathrm{mg} / \mathrm{g}$ and $180 \mathrm{mg} / \mathrm{g}$ respectively without impacting the overall quality of the oil. Mice in Group G6 were given the same EVSO as in G4 containing 120mg/g EPA+DHA but treated with heat at 190C for 5 minutes to simulate vacuum molecular distillation. $\left[{ }^{28}\right]$ Mice in Group G7 were given EVSO as in G4 but the EVSO was hydrolyzed with base and re-esterified with glycerol/acid as done in the standard fractional distillation step $\left.{ }^{29}\right]$ often carried out for producing EPA/DHA concentrated fish oils. Finally, the mice in Group G8 were given EVSO as in G4 but the EVSO had been oxidized (by air bubbling) to reach a totox of 24 which is at the upper limit of the GOED standard. [30]

Description of the ELISA Assay for oxLDL/32GPI concentrations: To a pre-prepped 96 micro well plate (Cayman Chemicals Inc., USA) $100 \mu \mathrm{L}$ of mouse serum samples diluted 1:100 in sample diluent was added (100 mmol/L tris(hydroxymethyl) aminomethane hydrochloride saline containing $2 \% \mathrm{BSA}$ and $5 \mathrm{mmol} / \mathrm{L}$ magnesium chloride, $\mathrm{pH} 8.5$ ) and allowed to incubate at room temperature for 1 hour. The micro wells were washed four times with phosphatebuffered saline containing $0.05 \%$ polysorbate-20 between each step. Biotinylated 2E10 anti-body (IgG murine monoclonal anti-mouse ApoB-100) diluted in $20 \mathrm{mmol} / \mathrm{L}$ HEPES-saline containing $2 \%$ BSA at $\mathrm{pH} 7.4$ was added to the micro wells and incubated for 30 minutes at room temperature, followed by horseradish peroxidase-streptavidin for 30 minutes. Color was developed with tetramethylbenzidine/hydrogen peroxide for 30 minutes and the reaction stopped with $0.3 \mathrm{~N}$ sulfuric acid. Optical density was read at a wavelength of $450 \mathrm{~nm}$. The functional sensitivity was $0.05 \mathrm{U} / \mathrm{ml}$. Serum oxLDL/B2GPI complex concentration (expressed in U/mL) was calculated against a reference curve generated from 3-fold serial dilutions of the reference serum. The specific interaction between oxidized LDL and $\beta 2$ GPI and the specificity of the assay for oxLDL/32GPI complexes has been previously reported.[31]

\section{RESULTS:}

No incidences of mortality were noted in any group during the 8 weeks of study period. No treatment related clinical signs or symptoms were observed in any of the animals, throughout the study period.

No significant difference was observed in the percentage body weight changes of treated animals as compared with the controls, throughout the treatment period.

Food consumption in animals of the treatment groups was comparable to the control group.

At gross necropsy, none of the animals of treatment groups showed any external or internal abnormal changes.

The eight treatment groups showed significantly different results based on both the total amount of daily EPA/DHA consumed as well as the different processing treatments carried out on the EVSO as shown in Table 3. Statistical analysis of each Group's results was carried out using the paired, one-tailed student t-test. 
Table 2. EVSO Specifications per Group

\begin{tabular}{|l|c|c|c|c|c|c|c|c|}
\hline COMPONENTS & G1 & G2 & G3 & G4 & G5 & G6 & G7 & G8 \\
\hline Saturated Fatty acids & 300 & 155 & 155 & 145 & 130 & 155 & 155 & 155 \\
\hline Monounsaturated fatty acids & 580 & 550 & 550 & 520 & 470 & 550 & 550 & 550 \\
\hline Omega-3 fatty acids & 20 & 160 & 160 & 200 & 255 & 155 & 160 & 160 \\
\hline Omega-6 fatty acids & 80 & 120 & 120 & 120 & 130 & 120 & 120 & 120 \\
\hline Eicosapentaenoic acid & 15 & 35 & 35 & 50 & 70 & 30 & 35 & 35 \\
\hline Docosapentaenoic acid & $<5$ & 15 & 15 & 15 & 15 & 15 & 15 & 15 \\
\hline Docosahexaenoic acid & 10 & 45 & 45 & 70 & 110 & 45 & 40 & 45 \\
\hline Asthaxanthin (mcg/gm) & $<1$ & 9 & 9 & 9 & 9 & 7 & 6 & 5 \\
\hline TOTOX & 8 & 12 & 12 & 12 & 12 & 11 & 15 & 27 \\
\hline
\end{tabular}

*All fatty acids assayed as methyl esters in $G C+/-5 m g / g$

Table 3. Differential EVSO Feeding impact on serum oxLDL- $\beta 2$-glycoprotein-I

\begin{tabular}{|c|c|c|c|c|c|c|c|}
\hline Group & $\begin{array}{l}\text { Mean Start } \\
\text { conc } \\
\text { (U/mol) }\end{array}$ & SD & $\begin{array}{l}\text { Mean End } \\
\text { conc (U/mol) }\end{array}$ & SD & $\begin{array}{l}\text { Percent } \\
\text { Reduction in } \\
\text { oxLDL-GP }\end{array}$ & P value & t value \\
\hline G1 & 4.964 & 0.3368 & 4.897 & 0.2959 & $-1.35 \%^{\mathrm{a}}$ & 0.3008 & 0.4944 \\
\hline G2 & 4.975 & 0.2605 & 4.712 & 0.2642 & $-5.29 \%^{\mathrm{b}}$ & 0.0326 & 2.0009 \\
\hline G3 & 5.037 & 0.4207 & 4.925 & 0.4234 & $-2.22 \%^{\mathrm{a}}$ & 0.3011 & 0.5331 \\
\hline G4 & 5.135 & 0.2274 & 4.851 & 0.3207 & $-5.53 \%^{\mathrm{b}}$ & 0.0381 & 1.9144 \\
\hline G5 & 5.062 & 0.2615 & 4.775 & 0.2659 & $-5.67 \%^{\mathrm{b}}$ & 0.0234 & 2.1802 \\
\hline G6 & 5.012 & 0.2357 & 4.787 & 0.2696 & $-4.49 \%^{\mathrm{b}}$ & 0.0488 & 1.7751 \\
\hline G7 & 5.125 & 0.2314 & 5.062 & 0.2825 & $-1.23 \%^{\mathrm{a}}$ & 0.3181 & 0.4836 \\
\hline G8 & 5.087 & 0.3137 & 4.925 & 0.3693 & $-3.18 \%^{\mathrm{a}}$ & 0.1796 & 0.9476 \\
\hline
\end{tabular}

$a=$ not statistically significant $; b=$ statistically significant $(P<0.05)$ 


\section{DISCUSSION:}

Chronic inflammatory diseases including atherosclerosis are a major cause of morbidity and mortality worldwide. Many studies as discussed above have shown the value of including oily fish in the diet for improved cardiovascular and cerebrovascular health[32] while other studies on fish oil supplements have shown mixed results. [33] A recent meta analysis of published studies[34] has even reported no correlation between omega-3 supplements and cardio-vascular health. Accumulating evidence suggests that certain oxidized low-density lipoprotein complexes, such as the serum circulating oxLDL- $\beta 2$-glycoprotein-I assayed here, represent endogenously formed factors that may be useful for diagnosing vascular inflammation, plaque build-up and CVD progression. In this study, we used this important CVD biomarker to further define the role of different components in extra virgin salmon oil in CVD treatment. By selectively increasing and decreasing the concentration of EPA+DHA without impacting the other oil components in any way, we were able to examine the specific effect these omega-3's had on modulating serum circulating oxLDL- $\beta 2$-glycoprotein-I.

Group G2 in Table 2 shows that a minimum intake of a human equivalent dose (HED) of 80 $\mathrm{mg} / \mathrm{day}$ of EPA+DHA is required for significant (95\% confidence; $\mathrm{P}<0.05$ ) lowering of serum concentrations of oxLDL-GP. At the same time, it is also apparent from the results of Groups G5 and G6 that further increases in EPA+DHA HED to $120 \mathrm{mg} / \mathrm{day}$ and $180 \mathrm{mg} / \mathrm{day}$ did not lead to additional statistically significant decreases in serum levels of oxLDL-GP.

Different processing treatment carried out on the EVSO also led to variable results. Heat treatment of the EVSO (Group G6) did not affect the ability of the oil to lower serum levels of oxLDL-GP. However, both acid/base hydrolysis followed by re-esterification to triglyceride esters (Group G7) and oxidative rancidity (Group G8), even to GOED acceptable fish oil capsule levels, eliminated the ability of the EVSO to reduce serum levels of oxLDL-GP, potentially making these processing treatments particularly detrimental to the ability of marine oils to benefit cardiovascular disease, as measured on the oxLDL-GP CVD biomarker.

One aspect of our results examines the effect of varying levels of EPA+DHA in nutraceutical marine oil intake ranging from HED of $40 \mathrm{mg} / \mathrm{day}$ to $180 \mathrm{mg} /$ day to reduce serum concentration levels of the new cardio-vascular biomarker, oxLDL-GP. The results showed that a minimum intake of $80 \mathrm{mg} / \mathrm{day}$ of EPA+DHA was necessary to show a statistically significant lowering of oxLDL-GP levels in serum but that increased intake did not significantly impact lowering of this critical CVD marker. This result is consistent with other results for minimum required omega-3 intakes for CVD effect such as reported by Albert et al [35] at 5g per month of omega-3 intake.

Another aspect of our results examined the effect of the various processing treatments on the ability of EVSO at reducing serum levels of oxLDL-GP at the same dose of EPA+DHA. We used the $120 \mathrm{mg}$ /day dose of EPA+DHA as the standard, and when subjected to $180 \mathrm{C}$ heat, the bioactivity of the EVSO was preserved but reduced (Group G6). However, upon treatment with acid or base and re-esterification to the triglyceride form (Group G7), the EVSO completely lost its bioactivity and the ability to reduce serum levels of oxLDL-GP. Similarly, upon oxidation with air to the limit of acceptable totox levels in commercial fish oil products (Group G8), the EVSO lost its ability to statistically significantly decrease serum levels of oxLDL-GP. Processing of foods and food ingredients often exerts a major effect on their constituents, 
especially the bioactive compounds. Liyana-Pathirana et al [36], reported that using different milling platforms changed the phenolic content of two wheat cultivars and this was reflected in free radical and reactive oxygen species (ROS) scavenging capacity and iron (II) chelation capacity of the products. Similarly processing of soybeans in the production of tofu greatly decreases the concentration of bioactive isoflavone compounds. [37] Our results are consistent with these reported losses of biological values in other overly processed foods.

\title{
CONCLUSION:
}

It may be concluded from our results that a minimum quantity of $80 \mathrm{mg} / \mathrm{day}$ HED of EPA+DHA omega- 3 acids is required to reduce the serum levels of oxLDL-GP and thus achieve a beneficial cardiovascular effect, but that increasing the amount of this daily intake is not a sufficient mechanism to further reduce circulatory oxLDL-GP serum levels. Instead, the presence of the maximum natural level of other lipid soluble bio-factors in minimally treated salmon oils (EVSO) seems to play a more important role in potentiating the effect of lowering oxLDL-GP serum concentrations, especially as observed for EVSO that has been subjected to harsh processing chemicals and solvents that reduce the presence of these bio-factors in the oil.

Our result on EVSO reinforce similar studies [38] on the same biomarker that show consuming a highly processed extract of a functional food does not result in the same quantitative biological effect as consuming the natural whole food or its minimally processed whole-food extract.

\section{Competing Interests:}

Bomi Framroze is the CSO of Hofseth Biocare where the EVSO was sourced. The other author has no financial interests or conflicts of interest.

\section{Authors' Contributions:}

Both authors, Bomi Framroze and Sagar Sawant contributed to this study.

\begin{abstract}
Abbreviations:
EVSO, extra virgin salmon oil; oxLDL-GP, oxidized low density lipoprotein $\beta 2$-glycoprotein I; CVD, cardio vascular disease; EPA, eicosapentenoic acid; DHA, docosahexenoic acid
\end{abstract}

\section{REFERENCES:}

1. Krauss, R., Eckel, R., Howard, B. AHA Dietary Guidelines: revision 2000: a statement for healthcare professionals from the Nutrition Committee of the American Heart Association. Circulation 102 (2000), pp. 2284-2299.

2. Zhang, J., Sasaki, S., Amano, K. Fish consumption and mortality from all causes, ischemic heart disease, and stroke: an ecological study. Prev Med. 28 (1999), pp. 520529

3. Daviglus, M., Stamler, J., Orencia, A. Fish consumption and the 30-year risk of fatal myocardial infarction. New England Journal of Medicine 336 (1997), pp. 1046-1053 
4. Kromhout, D., Bosschiete, E., de Lezenne Coulander, C. The inverse relation between fish consumption and 20-year mortality from coronary heart disease. New England Journal of Medicine 312 (1985), pp. 1205-1209

5. Kromhout, D., Feskens, E., Bowles, C. The protective effect of a small amount of fish on coronary heart disease mortality in an elderly population. Int J Epidemiol. 24 (1995), pp. 340-345

6. Hu, F., Bronner, L., Willett, W. Fish and omega-3 fatty acid intake and risk of coronary heart disease in women. JAMA 287 (2002), pp. 1815-1821

7. Siscovick, D., Raghunathan, T., King, I. Dietary intake and cell membrane levels of long-chain n-3 polyunsaturated fatty acids and the risk of primary cardiac arrest. JAMA 274 (1995), pp. 1363-1367

8. Ascherio, A., Rimm, E., Stampfer, M. Dietary intake of marine n-3 fatty acids, fish intake, and the risk of coronary disease among men. New England Journal of Medicine 332 (1995), pp. 977-982

9. Albert, C., Hennekens, C., O’Donnell, C. Fish consumption and risk of sudden cardiac death. JAMA 279 (1998), pp. 23-28

10. Guallar, E., Aro, A., Jimenez, F. Omega-3 fatty acids in adipose tissue and risk of myocardial infarction: the EURAMIC study. Arterioscler Thromb Vasc Biol. 19 (1999), pp. 1111-1118

11. Pietinen, P., Ascherio, A., Korhonen, P. Intake of fatty acids and risk of coronary heart disease in a cohort of Finnish men: the Alpha-Tocopherol, Beta-Carotene Cancer Prevention Study. Am J Epidemiol. 145 (1997), pp. 876-887

12. Sheard, N. Fish consumption and risk of sudden cardiac death. Nutr Rev. 56 (1998), pp. $177-179$

13. Marckmann, P., Grønbæk, M. Fish consumption and coronary heart disease mortality: a systematic review of prospective cohort studies. Eur J Clin Nutr. 53 (1999), pp. 585-590

14. Oomen, C., Feskens, E., Rasanen, L. Fish consumption and coronary heart disease mortality in Finland, Italy, and The Netherlands. Am J Epidemiol. 151 (2000), pp. 9991006

15. Rissanen, T., Voutilainen, S., Nyyssonen, K. Fish oil-derived fatty acids, docosahexaenoic acid and docosapentaenoic acid, and the risk of acute coronary events: the Kuopio ischaemic heart disease risk factor study. Circulation. 102 (2000), pp. 26772679

16. Ascherio, A., Rimm, E., Giovannucci, E. Dietary fat and risk of coronary heart disease in men: cohort follow up study in the United States. BMJ. 313 (1996), pp. 84-90

17. Visioli F, Galli C. Alpha-linolenic acid and cardiovascular disease. Am J Clin Nutr. 75 (2002), pp. 1121

18. De Lorgeril, M., Salen, P., Martin, J. Mediterranean diet, traditional risk factors, and the rate of cardiovascular complications after myocardial infarction: final report of the Lyon Diet Heart Study. Circulation. 99 (1999), pp. 779-785 
19. Gruppo Italiano per lo Studio della Sopravvivenza nell'Infarto miocardico. Dietary supplementation with n-3 polyunsaturated fatty acids and vitamin E after myocardial infarction: results of the GISSI-Prevenzione trial. Lancet. 354 (1999), pp. 447-455

20. Folsom, A., Chambless, L., Ballantyne, C. An assessment of incremental coronary risk prediction using C-reactive protein and other novel risk markers. Arch Intern Med 166 (2006), pp. 1368-1373

21. Saifullah, A., Watkins, B., Saha, C., Li, Y., Moe, S., Friedman, A. Oral fish oil supplementation raises blood omega-3 levels and lowers C-reactive protein in haemodialysis patients: a pilot study. Nephrology Dialysis Transplantation 22 (2007), pp. 3561-3567

22. Meisinger, C., Baumert, J., Khuseyinova, N. Plasma oxidized low-density lipoprotein, a strong predictor for acute coronary heart disease events in apparently healthy, middleaged men from the general population. Circulation 112 (2005), pp. 651-657

23. Damsgaard, C., Frokiaer, H., Andersen, A., Lauritzen, L. Fish Oil in Combination with High or Low Intakes of Linoleic Acid Lowers Plasma Triacylglycerols but Does Not Affect Other Cardiovascular Risk Markers in Healthy Men. Journal of Nutrition 138 (2008), pp. 1061-1066.

24. Kobayashi, K., Matsuura, E., Liu, Q. A specific ligand for $\beta 2$-glycoprotein I mediates autoantibody-dependent uptake of oxidized low-density lipoproteins by macrophages. J Lipid Res 42 (2001), pp. 697-709

25. Greco, T., Conti-Kelly, A., Anthony, R. Oxidized-LDL/ 2-Glycoprotein I Complexes Are Associated With Disease Severity and Increased Risk for Adverse Outcomes in Patients With Acute Coronary Syndromes. Am J of Clin Pathlogy 133 (2010), pp. 737743

26. Framroze, B., Sen, N. The Effect of Dietary Oil Capsules on Reducing Serum Concentrations of Oxidized Low Density Lipoprotein- $\beta 2$-Glycoprotein-I Complex. J Nutr Food Sci 3:5 (2013) 225-229

27. Kahveci, D., Falkeborg M., Gregersen, S., and Xu, X. Upgrading of Farmed Salmon Oil Through Lipase-Catalyzed Hydrolysis. The Open Biotechnology Journal 4 (2010), pp. 47-55

28. Oliveira, A., Miller, M. Purification of Alaskan Walleye Pollock (Gadus chalcogrammus) and New Zealand Hoki (Macruronus novaezelandiae) Liver Oil Using Short Path Distillation. Nutrients 6(5) (2014), pp. 2059-2076

29. Tengku-Rozaina, T., Birch, J. Enrichment of Omega-3 Fatty Acids of Refined Hoki Oil. Journal of the American Oil Chemists' Society 90(8) (2013), pp. 1111-1119

30. Global Organization for EPA and DHA Omega-3 (GOED) Voluntary Monograph. Available online: http://www.goedomega3.com/images/stories/files/goedmonograph.pdf

31. Lopez, D., Garcia-Valladares, I., Palfox-Sanchez, C. Oxidized low-density/ $/ 2$ glycoprotein I complexes and autoantibodies to oxLig-1/32-glycoprotein I in patients with systemic lupus erythematosus and antiphospholipid syndrome. Am J Clin Pathol 121 (2004), pp. 426-436. 
32. Franco, O., Chowdhury, R., Stevens, S., Gorman, D., Pan, A. et al. Association between fish consumption, long chain omega-3 fatty acids and risk of cerebrovascular disease; systematic review and meta anaylsis. British Medical Journal 345 (2012), pp. e6698

33. Kromhout, D., Yasuda, S., Geleijnse, J., Shimokawa, H. Fish oil and omega-3 fatty acids in cardiovascular disease: do they really work. European Heart Journal 33 (2012), pp. 436-443

34. Rizos, E., Ntzani, E., Bika, E., Kostapanos, M., Elisaf, M. Association Between Omega3 Fatty Acid Supplementation and Risk of Major Cardiovascular Disease Events. A Systematic Review and Meta-analysis. JAMA 308(10) 2013, pp. 1024-1033

35. Albert, M., Campos, H., Stampfer, M. Blood levels of long-chain n-3 fatty acids and the risk of sudden death. N Engl J Med. 346 (2002), pp. 1113-1118

36. Liyana-Pathirana, C., Shahidi, F. The antioxidant potential of milling fractions from breadwheat and durum. Journal of Cereal Science 45 (2007), pp. 238-247

37. Jackson, C., Dini, J., Lavandier, C., Rupasinghe, H., Faulkner, H., Poysa, V. Effects of processing on the content and composition of isoflavones during manufacturing of soy beverage and tofu. Process Biochemistry 37 (2002), pp. 1117-1123

38. Zhao, S., Bomser, J., Joseph, E., DiSilvestro, R. Intakes of apples or apple polyphenols decease plasma values for oxidized low-density lipoprotein/beta2-glycoprotein I complex. Journal of Functional Foods 5(1) 2013, pp. 493-497 